\title{
Prävention sinnvoll
}

\section{Kinderkriegen verstärkt Sportmuffelei}

Hull EE, Rofey DL, Robertson RJ et al. J Phys Act Health 2010;7(5):577-83.

\section{P, Iverson DC. J}

Magee CA, Caputi

Behav Med 2011;

34(2):83-91.

\section{Das Lifestyle-Telegramm zu} hochwertigen Publikationen mit dem Thema "Lebensstil" kann als Newsletter kostenlos abonniert werden und erscheint monatlich per Mail. Viele weitere entsprechende Kurzreferate gibt's unter: www.lifestyle-telegramm.de
Wenn Jugendliche zu jungen Erwachsenen werden, sinkt oft ihre körperliche Aktivität. Als mögliche Gründe hierfür galten bisher sogenannte „Lebensübergänge“. Mithilfe einer Studie sollten daher Lebensübergänge wie die Heirat oder die Geburt eines Kindes und deren Auswirkungen auf die Menge an körperlicher Aktivität bei jungen Erwachsenen untersucht werden.

Dazu wurden mit Fragebögen über zwei Jahre prospektiv die körperliche Aktivität (in Stunden pro Woche) sowie sonstige soziodemografischen Faktoren bei 638 jungen Erwachsenen dokumentiert (48\% Männer, 15\% farbige Menschen, Durchschnittsalter 24 +/- 1,1 Jahre). Die Angaben zur körperlichen Aktivität wurden log- transformiert und mit Kovarianzanalysen (ANCOVAs) untersucht.

Ergebnisse: Nach einer Hochzeit änderte sich die Menge an körperlicher Aktivität im Vergleich zu weiterhin unverheirateten Personen nicht signifikant $[F(1,338)=0,38, p=0,54, d=0,06]$. Nach der Geburt eines Kindes verringerte sich die Menge an körperlicher Aktivität jedoch signifikant im Vergleich zu weiterhin kinderlosen Testpersonen $[\mathrm{F}(1,517)=6,7, \mathrm{p}=0,01, \mathrm{~d}=0,41]$. Dies zeigt, dass entsprechende Interventionsprogramme bei jungen Eltern besonders sinnvoll wären - wenn sie Zeit dafür finden.

\section{Erhöhter Body-Mass-Index}

\section{Viel Arbeit, wenig Schlaf und deshalb dick?}

Es sollte untersucht werden, inwieweit eine kurze Schlafdauer, körperliche Aktivität und die täglich sitzend verbrachte Zeit bei der Assoziation zwischen langen Arbeitszeiten und dem Body Mass Index (BMI) eine Rolle spielen. An der Studie nahmen 16951 Personen mittleren Alters aus Australien teil, die vollzeitbeschäftigt waren und mindestens 35 Stunden pro Woche arbeiteten. Anhand von Fragebögen machten die Teilnehmer eigene Angaben zum BMI, zur Schlafdauer und zur Arbeitszeit sowie zu sonstigen gesundheitsbezogenen und demografischen Faktoren. In einem multiplen Mediationsmodell wurden die Schlafdauer, die körperliche Aktivität sowie die sitzend verbrachte Zeit als mögliche Mediatoren zwischen der Arbeitszeit und dem BMI eingegeben.

Ergebnisse: Eine kurze Schlafdauer führt bei Männern z.T. zu einer Assoziation zwischen langen Arbeitszeiten und erhöhtem BMI. Bei Frauen führte wenig Schlaf nur indirekt zu diesem Zusammenhang.

Insgesamt belegen die Ergebnisse in gewissem Maß die Hypothese, dass eine lange Arbeitszeit durch eine Verringerung der Schlafdauer zu Fettleibigkeit führen kann, weshalb weitere prospektive Studien zu dieser Frage erforderlich sind.

\section{Fernsehkonsum}

\section{Männern geht er auch an die Nieren}

Lynch BM, White $\mathrm{SL}$, Owen $\mathrm{N}$ et al. Ann Behav Med 2010;40(3):265-74.
Die Menge des Fernsehkonsums wird unabhängig von der jeweiligen körperlichen Aktivität mit einer Reihe chronischer Erkrankungen sowie den zugehörigen Risikofaktoren assoziiert. Es ist jedoch nicht bekannt, welcher Zusammenhang zwischen der Fernsehdauer und chronischen Nierenerkrankungen besteht. Mit Hilfe der vorliegenden prospektiven Querschnittsstudie sollte daher untersucht werden, in welchem Zusammenhang die Fernsehdauer mit Biomarkern chronischer Nierenerkrankungen steht. Dazu wurden im Rahmen der „Australian Diabetes, Obesity and Lifestyle“-Studie zunächst 10847 Teilnehmer sowie nach einer 5-jährigen Beobachtungszeit 6293 Teilnehmer untersucht.
Ergebnisse: Die Fernsehdauer war bei Männern, nicht jedoch bei Frauen, signifikant mit einem erhöhten Risiko für eine Eiweißausscheidung im Urin (Albuminurie) sowie mit einer verringerten Nierenfunktion (berechnete glomeruläre Filtrationsrate, GFR) assoziiert. Eine Langzeitanalyse ergab, dass das Risiko des Neuauftretens von Albuminurie sowie einer erniedrigten GFR nur bei nicht adjustierten Modellen erhöht war. Somit kann die Fernsehdauer sowohl direkt als auch durch entsprechende Risikofaktoren wie Diabetes, Bluthochdruck und Fettleibigkeit mit Markern chronischer Nierenerkrankungen in Zusammenhang gebracht werden. Quelle (3 Texte): www.lifestyle-telegramm.de 